\title{
On the electromagnetic force on a polarizable body
}

\author{
A. Engel ${ }^{a)}$ and R. Friedrichs, \\ Institut für Theoretische Physik, Otto-von-Guericke-Universität Magdeburg, PSF 4120, 39016 \\ Magdeburg, Germany
}

The force on a macroscopic polarizable body in an inhomogenous electromagnetic field is calculated for three simple exactly solvable situations. Comparing different approaches we pinpoint possible pitfalls and resolve recent confusion about the force density in ferrofluids.

\section{Introduction}

The force on a macroscopic electrically or magnetically polarizable body in an external electromagnetic field is one of the most basic and at the same time most relevant problems in classical field theory. Although all texts ${ }^{1-4}$ on the subject introduce Maxwell stress and the force on a (microscopic) dipol there is still considerable confusion on how to use these concepts in concrete situations. That these problems are not confined to the classroom is exemplified by a recent controversy among experts on the magnetic force density in ferrofluids 5,6 .

In order to discuss the possible problems which may arise when calculating the force on a polarizable body it is very useful to study simple and exactly solvable cases. Since a non-zero force requires an inhomogenous external field such examples are not abound. The purpose of the present paper is to present three simple situations in thermodynamic equilibrium for which fields, stresses and forces can all be calculated exactly, using only basic Maxwell theory, and to discuss for these situations different methods to calculate the resulting ponderomotive forces. The first example will be dealt with in detail whereas for the remaining two only the main results will be given.

\section{Basic equations}

Neglecting effects of electro- or magnetostriction the Maxwell stress tensor for a macroscopic polarizable medium in equilibrium is of the form ${ }^{2,4}$

$$
T_{i j}=-\frac{1}{2}\left(\varepsilon_{0} \mathbf{E}^{2}+\mu_{0} \mathbf{H}^{2}\right) \delta_{i j}+E_{i} D_{j}+H_{i} B_{j},
$$

where as usual $\mathbf{E}$ denotes the electric field, $\mathbf{D}$ the electric displacement, $\mathbf{H}$ the magnetic field, and $\mathbf{B}$ the magnetic induction. From this stress tensor one finds the Kelvin expression

$$
f_{i}:=\partial_{j} T_{i j}=\left(P_{j} \partial_{j}\right) E_{i}+\mu_{0}\left(M_{j} \partial_{j}\right) H_{i}
$$

for the ponderomotive force density in the medium. Here $\mathbf{P}:=\mathbf{D}-\varepsilon_{0} \mathbf{E}$ denotes the electric polarization and $\mathbf{M}:=\mathbf{B} / \mu_{0}-\mathbf{H}$ the magnetization of the medium whereas $\varepsilon_{0}$ and $\mu_{0}$ are the dielectric constant and magnetic permeability respectively of the vacuum. Finally the change of the equilibrium free energy of a polarizable body due to a change of the fields is given by ${ }^{3,4}$

$$
\delta F=-\int_{V} d^{3} r\left(\mathbf{P} \cdot \delta \mathbf{E}_{0}+\mu_{0} \mathbf{M} \cdot \delta \mathbf{H}_{0}\right)
$$


where the integral is over the volume of the body, and $\mathbf{E}_{0}$ and $\mathbf{H}_{0}$ denote the external fields in the absence of the polarizable material.

All three expression are consistent with each other and can therefore be used equally well to determine the total force on a macroscopic body. In the following examples we will always assume linear constitutive relations, i.e. $\mathbf{D}=\varepsilon_{0} \varepsilon_{r} \mathbf{E}$ and $\mathbf{B}=\mu_{0} \mu_{r} \mathbf{H}$, since we are only concerned with effects for which a possible nonlinearity in this relations is not crucial.

\section{Point charge polarizing a half space}

Let us first consider a point charge $Q$ at $\boldsymbol{a}=(0,0, a)$ a distance $a>0$ away from a dielectric half space with relative dielectric constant $\varepsilon_{r}>1$ (see fig.11). We will neglect possible magnetic effects and study only the electric part of the problem.

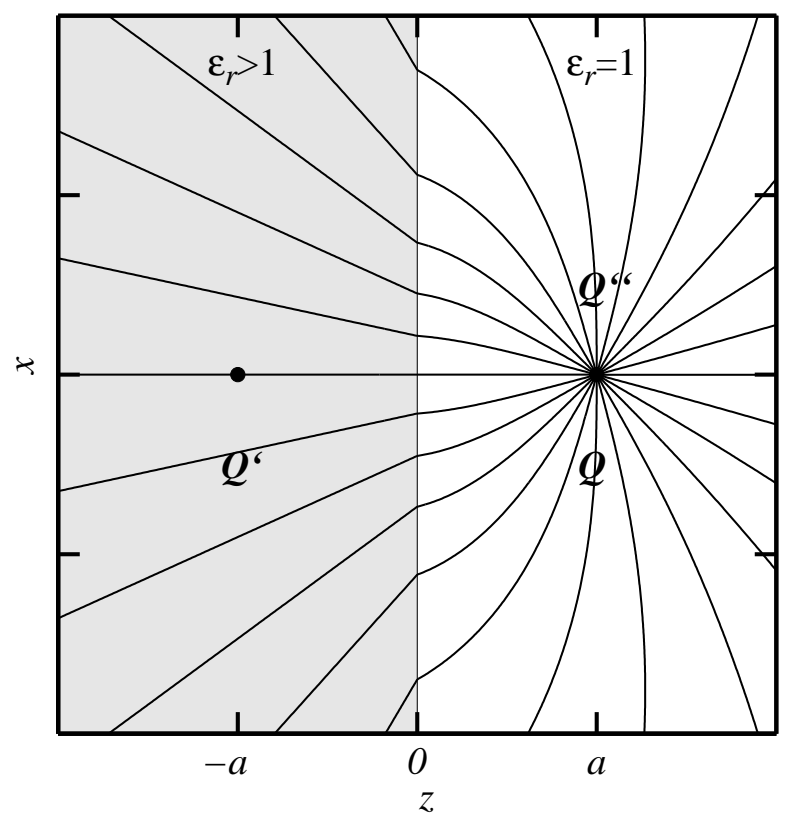

Figure 1: Lines of the electric displacement field $\mathbf{D}$ for a point charge $Q$ polarizing a dielectric half space with $\varepsilon_{r}=3$. The field is symmetric with respect to rotations around the $z$-axis.

As is well known ${ }^{2,1}$ the electrostatic problem can be solved exactly with the help of a mirror charge $Q^{\prime}=-Q\left(\varepsilon_{r}-1\right) /\left(\varepsilon_{r}+1\right)$ at $\boldsymbol{r}=-\boldsymbol{a}$ and an additional auxiliary charge $Q^{\prime \prime}=2 Q /\left(\varepsilon_{r}+1\right)$ at $\boldsymbol{r}=\boldsymbol{a}$. Using the coordinate system of fig.1 the result for the electric field is

$$
\mathbf{E}(\boldsymbol{r})= \begin{cases}\frac{Q}{4 \pi \varepsilon_{0}} \frac{2}{\varepsilon_{r}+1} \frac{\boldsymbol{r}-\boldsymbol{a}}{|\boldsymbol{r}-\boldsymbol{a}|^{3}} & \text { if } \quad z \leq 0 \\ \frac{Q}{4 \pi \varepsilon_{0}}\left[\frac{\boldsymbol{r}-\boldsymbol{a}}{|\boldsymbol{r}-\boldsymbol{a}|^{3}}-\frac{\varepsilon_{r}-1}{\varepsilon_{r}+1} \frac{\boldsymbol{r}+\boldsymbol{a}}{|\boldsymbol{r}+\boldsymbol{a}|^{3}}\right] & \text { if } z \geq 0\end{cases}
$$

Our aim is to determine the force $\mathbf{F}$ with which the point charge attracts the half space. 


\subsection{The physical picture}

The field of the point charge polarizes the dielectric which results in an induced bulk charge density $\rho=-\nabla \mathbf{P}$ and a surface charge density $\sigma=P_{n}:=\mathbf{P} \cdot \boldsymbol{n}$ where $\mathbf{P}=\varepsilon_{0}\left(\varepsilon_{r}-1\right) \mathbf{E}$ is the polarization of the medium and $\boldsymbol{n}$ denotes the normal vector on the surface pointing outward of the medium. In our case $\boldsymbol{n}=(0,0,1)$. Since the field in the dielectric is a Coulomb field of a point charge (cf.(A)) we have $\nabla \mathbf{P}=0$ and no bulk charge density is induced. For the surface charge we find from (4) setting $z=0$

$$
\sigma(x, y)=-\varepsilon_{0}\left(\varepsilon_{r}-1\right) \frac{Q}{4 \pi \varepsilon_{0}} \frac{2}{\varepsilon_{r}+1} \frac{a}{\left(x^{2}+y^{2}+a^{2}\right)^{3 / 2}} .
$$

Summing up the Coulomb forces between $Q$ and the elements of this surface charge we find $\mathbf{F}=(0,0, F)$ with

$$
F=\frac{Q^{2}}{4 \pi \varepsilon_{0}} \frac{\varepsilon_{r}-1}{\varepsilon_{r}+1} \frac{1}{4 a^{2}} .
$$

This is an unambigous and physically sound expression for the force in question. It is exactly equal to the Coulomb force between $Q$ and the mirror charge $Q^{\prime}$.

\subsection{Integrating the Kelvin force}

For the Kelvin force density (2) we find in the present situation

$$
\mathbf{f}=\frac{\varepsilon_{0}}{2}\left(\varepsilon_{r}-1\right) \nabla \mathbf{E}^{2}=-\frac{Q^{2}}{\left(4 \pi \varepsilon_{0}\right)^{2}} \frac{8 \varepsilon_{0}\left(\varepsilon_{r}-1\right)}{\left(\varepsilon_{r}+1\right)^{2}} \frac{\boldsymbol{r}-\boldsymbol{a}}{|\boldsymbol{r}-\boldsymbol{a}|^{6}} .
$$

Integrating this expression over the halfspace $z<0$ the $x$ and $y$ components again average to zero and for the $z$ component we get

$$
F^{\text {Kelvin }}=\frac{Q^{2}}{4 \pi \varepsilon_{0}} \frac{2\left(\varepsilon_{r}-1\right)}{\left(\varepsilon_{r}+1\right)^{2}} \frac{1}{4 a^{2}} .
$$

This is clearly different from (6): the integral over the Kelvin force density does not yield the total force on a polarizable body. This is what was observed also in ${ }^{5}$ for another example. The reason for this discrepancy is however not that the expressions (2) or (7) for the Kelvin force density are incorrect but that the total electromagnetic force on the polarizable medium involves both bulk and surface contributions whereas (8) only covers the bulk part. The expression for the appropriate surface part is given below.

\subsection{Balance of stresses}

By the definition of stress the total force on a body is given by the integral of the stress tensor over the complete surface of the body. Paying attention to the fact that the Maxwell stress is non-zero also outside the polarizable medium a calculation of the electromagnetic force on a body has to integrate the differences in stress slightly above and below the surface of the body. In our particular problem the fields decay sufficiently rapidly to zero for $r \rightarrow \infty$ such that only the integral over the $x$-y-plane contributes. Using (11) and (4) we find for the relevant component of the Maxwell stress tensor

$$
\lim _{z \rightarrow-0} T_{z z}(x, y, z)=\frac{Q^{2}}{\left(4 \pi \varepsilon_{0}\right)^{2}} \frac{2 \varepsilon_{0}}{\left(\varepsilon_{r}+1\right)^{2}}\left[\frac{2 \varepsilon_{r} a^{2}}{\left(x^{2}+y^{2}+a^{2}\right)^{3}}-\frac{1}{\left(x^{2}+y^{2}+a^{2}\right)^{2}}\right]
$$


on the medium side and

$$
\lim _{z \rightarrow+0} T_{z z}(x, y, z)=\frac{Q^{2}}{\left(4 \pi \varepsilon_{0}\right)^{2}} \frac{2 \varepsilon_{0}}{\left(\varepsilon_{r}+1\right)^{2}}\left[\frac{\varepsilon_{r}^{2} a^{2}}{\left(x^{2}+y^{2}+a^{2}\right)^{3}}-\frac{x^{2}+y^{2}}{\left(x^{2}+y^{2}+a^{2}\right)^{3}}\right]
$$

on the vacuum side. Integrating the difference between these electric stresses one finds for the $z$ component of the force

$$
F^{\text {electric }}=\frac{Q^{2}}{4 \pi \varepsilon_{0}}\left(\frac{\varepsilon_{r}-1}{\varepsilon_{r}+1}\right)^{2} \frac{1}{4 a^{2}} .
$$

This is again different from the correct expression (6)! The reason for this renewed failure is somewhat more subtle. The point is that we have only considered the electric part of the stress tensor in the medium whereas the total force must clearly be related to the surface integral of the total stress tensor $T_{i j}^{\text {total }}$. But in equilibrium we must have $\partial_{j} T_{i j}^{\text {total }}=0$ (otherwise there would be momentum transport in the medium) and the electric part (9) is hence counterbalanced by non-electric contributions. If our dielectric is a solid, elastic stresses would build up, if it is a fluid a non-trivial pressure field would compensate the electric stress. In any case the total internal stress in equilibrium must be divergence free and hence cannot contribute to the total force on the body.

The whole stress on the surface is therefore given by the vacuum part (10) and its surface integral indeed yields the correct result (6). Incidentally this also explains why the force is equal to the Coulomb force between $Q$ and $Q^{\prime}$ : the field for $z>0$ is identical for either the polarizable half space or the dielectric replaced by the mirror charge $Q^{\prime}$ and hence the integrals over the vacuum stress must be the same.

Let us finally note that from the definition $f_{i}:=\partial_{j} T_{i j}$ of the Kelvin force density it is clear that the volume integral of $f_{i}$ must be identical to the surface integral of $T_{i j}$. Hence the elimination of the contribution from the electric stress inside the medium in (11) can simply be accomplished by adding (8). Equivalently (11) is nothing but the surface contribution that was missed in (8). Using the behaviour of the fields $\mathbf{E}$ and $\mathbf{D}$ at the interface between media with different dielectric constant it is easy to show ${ }^{4}$ that

$$
n_{i}\left(T_{i j}^{\text {vacuum }}-T_{i j}^{\text {medium }}\right) n_{j}=\frac{1}{2 \varepsilon_{0}}(\mathbf{P} \cdot \boldsymbol{n})^{2} .
$$

Hence the appropriate surface force density complementing the Kelvin body force density is given by

$$
\mathbf{f}^{\text {surface }}=\frac{1}{2 \varepsilon_{0}} \mathbf{P}_{n}^{2} \boldsymbol{n}
$$

\subsection{Change in free energy}

The simplest and most direct way to determine the total force on a polarizable body in equilibrium is to use expression (3) for the variation in the free energy. Specializing to the case without magnetic contributions we note first that the change in the electric field $\mathbf{E}_{0}$ in the region of the body due to a displacement of the body by an infinitesimal vector $\delta \boldsymbol{r}$ is given by $\delta \mathbf{E}_{0}=(\delta \boldsymbol{r} \cdot \nabla) \mathbf{E}_{0}$. On the other hand the corresponding change in free energy is related to the force $\mathbf{F}$ via $\delta F=-\mathbf{F} \cdot \delta \boldsymbol{r}$ by the very definition of the total force $\mathbf{F}$. Using the condition $\nabla \times \mathbf{E}_{0}=0$ fulfilled in thermodynamic equilibrium we therefore find

$$
\mathbf{F}=\int_{V} d^{3} r(\mathbf{P} \cdot \nabla) \mathbf{E}_{0} .
$$


Using (14) to determine $\mathbf{P}$ as well as

$$
\mathbf{E}_{0}(\boldsymbol{r})=\frac{Q}{4 \pi \varepsilon_{0}} \frac{\boldsymbol{r}-\boldsymbol{a}}{|\boldsymbol{r}-\boldsymbol{a}|^{3}}
$$

and performing the integral we indeed recover the correct expression (6).

\section{Charged line polarizing a parallel cylinder}

This is again an example from electrostatics. A line at distance $a$ from and parallel to the $y$-axis carries a charge $Q$ per length. A dielectric cylinder of radius $R$, dielectric constant $\varepsilon_{r}$, and the $y$-axis as symmetry axis is polarized by the field of the line charge (see fig.2). We want to determine the force with which the cylinder is attracted to the line.

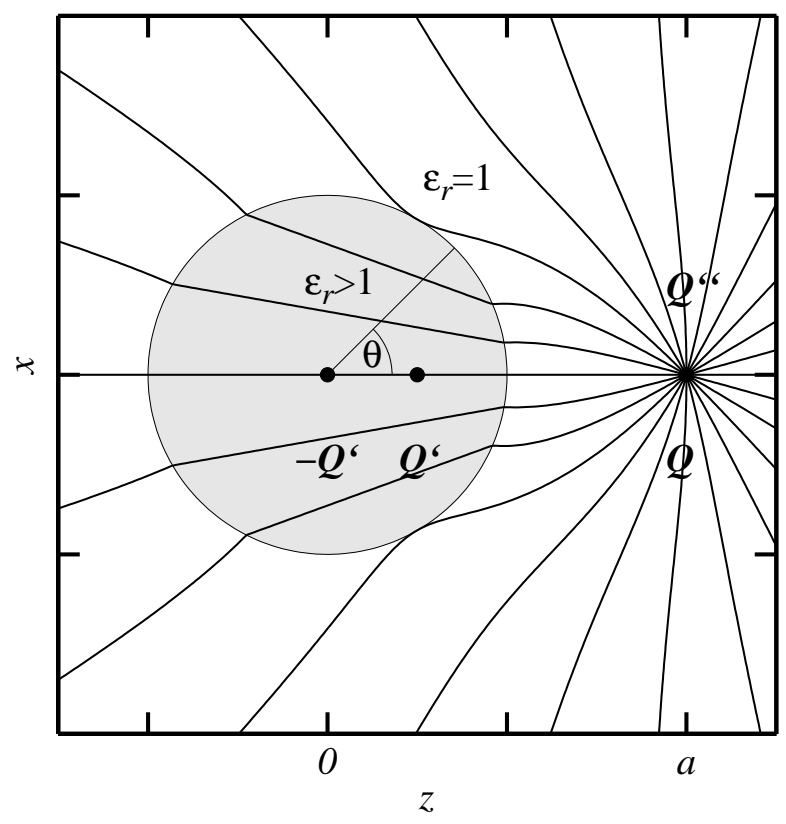

Figure 2: Lines of the electric displacement field $\mathbf{D}$ for a charged line polarizing a parallel dielectric cylinder with $\varepsilon_{r}=3$ and radius $R=a / 2$, where $a$ denotes the distance between the axis of the cylinder and the charged line. The situation is symmetric with respect to translations in the $y$-direction.

Due to the translational invariance along the $y$-axis the problem is essentially twodimensional and accordingly we use the notation $\boldsymbol{r}=(x, 0, z)$ in the following. The electrostatic problem can again be solved by the method of images. To determine the fields outside the dielectric one introduces two additional charged lines with densities $Q^{\prime}=-Q\left(\varepsilon_{r}-\right.$ $1) /\left(\varepsilon_{r}+1\right)$ at a distance $a^{\prime}:=R^{2} / a$ from the $y$-axis and $-Q^{\prime}$ at the $y$-axis respectively. The field inside the dielectric is determined by replacing the original line by one with charge 
density $Q^{\prime \prime}=2 Q /\left(\varepsilon_{r}+1\right)$. The resulting electric field is given by

$$
\mathbf{E}(\boldsymbol{r})=\left\{\begin{array}{ll}
\frac{Q}{2 \pi \varepsilon_{0}} \frac{2}{\varepsilon_{r}+1} \frac{\boldsymbol{r}-\boldsymbol{a}}{|\boldsymbol{r}-\boldsymbol{a}|^{2}} & \text { if } \quad r \leq R \\
\frac{Q}{2 \pi \varepsilon_{0}}\left[\frac{\boldsymbol{r}-\boldsymbol{a}}{|\boldsymbol{r}-\boldsymbol{a}|^{2}}-\frac{\varepsilon_{r}-1}{\varepsilon_{r}+1} \frac{\boldsymbol{r}-\boldsymbol{a}^{\prime}}{\left|\boldsymbol{r}-\boldsymbol{a}^{\prime}\right|^{2}}+\frac{\varepsilon_{r}-1}{\varepsilon_{r}+1} \frac{\boldsymbol{r}}{r^{2}}\right] & \text { if } \quad r \geq R
\end{array} .\right.
$$

The field inside the dielectric is again divergence free. The induced surface charge can be determined from the normal component of the polarization as in the first example and is found to be

$$
\sigma(\theta)=\frac{Q}{\pi} \frac{\varepsilon_{r}-1}{\varepsilon_{r}+1} \frac{R-a \cos \theta}{R^{2}-2 R a \cos \theta+a^{2}},
$$

where $\theta$ denotes the angle between the radius vector to the surface element in question and the $z$-axis. Using the two-dimensional Coulomb law and summing up the $z$-components of the forces between the surface elements and the original charged line we find

$$
F=\frac{Q^{2}}{2 \pi \varepsilon_{0}} \frac{\varepsilon_{r}-1}{\varepsilon_{r}+1} \frac{R^{2}}{a\left(a^{2}-R^{2}\right)} .
$$

As expected this is exactly the sum of the Coulomb forces between $Q, Q^{\prime}$ and $-Q^{\prime}$.

The most direct way to calculate this force is to use (3) in the form (14). Since $\mathbf{P}$ is parallel to $\mathbf{E}_{0}$ we have

$$
(\mathbf{P} \nabla) \mathbf{E}_{0}=P \nabla E_{0}=-\frac{Q^{2}}{2 \pi^{2} \varepsilon_{0}} \frac{\varepsilon_{r}-1}{\varepsilon_{r}+1} \frac{\boldsymbol{r}-\boldsymbol{a}}{|\boldsymbol{r}-\boldsymbol{a}|^{4}} .
$$

Integrating this expression over the volume of the dielectric cylinder the $x$-component averages to zero whereas the $z$-component yields back (18).

\section{Magnetizable cylinder in the field of a straight wire}

Finally we discuss a simple magnetic example for which the electric part will be neglected. Consider a straight wire at distance $a$ from the $y$-axis carrying a current $I$. A cylinder of radius $R$ and magnetic permeability $\mu_{r}$ with the $y$-axis as symmetry axis is placed into the magnetic field of the wire. We want to determine the force per length on the cylinder.

Due to the well known similarities between the magnetic fields of parallel, stationary currents and two-dimensional electrostatics the algebra for this example is very similar to the previous one. We introduce two mirror currents, $I^{\prime}=I\left(\mu_{r}-1\right) /\left(\mu_{r}+1\right)$ parallel to the original one at a distance $a^{\prime}=R^{2} / a$ from the $y$-axis and $-I^{\prime}$ along the $y$-axis. The field inside the medium is given by replacing $I$ by $I^{\prime \prime}=2 I /\left(\mu_{r}+1\right)$. The magnetic field is then found to be

$$
\mathbf{H}(\boldsymbol{r})=\left\{\begin{array}{ll}
\frac{I}{2 \pi} \frac{2}{\mu_{r}+1} \frac{\boldsymbol{e}_{y} \times(\boldsymbol{r}-\boldsymbol{a})}{|\boldsymbol{r}-\boldsymbol{a}|^{2}} & \text { if } \quad r \leq R \\
\frac{I}{2 \pi}\left[\frac{\boldsymbol{e}_{y} \times(\boldsymbol{r}-\boldsymbol{a})}{|\boldsymbol{r}-\boldsymbol{a}|^{2}}+\frac{\mu_{r}-1}{\mu_{r}+1} \frac{\boldsymbol{e}_{y} \times\left(\boldsymbol{r}-\boldsymbol{a}^{\prime}\right)}{\left|\boldsymbol{r}-\boldsymbol{a}^{\prime}\right|^{2}}-\frac{\mu_{r}-1}{\mu_{r}+1} \frac{\boldsymbol{e}_{y} \times \boldsymbol{r}}{|\boldsymbol{r}|^{2}}\right] & \text { if } \quad r \geq R
\end{array},\right.
$$




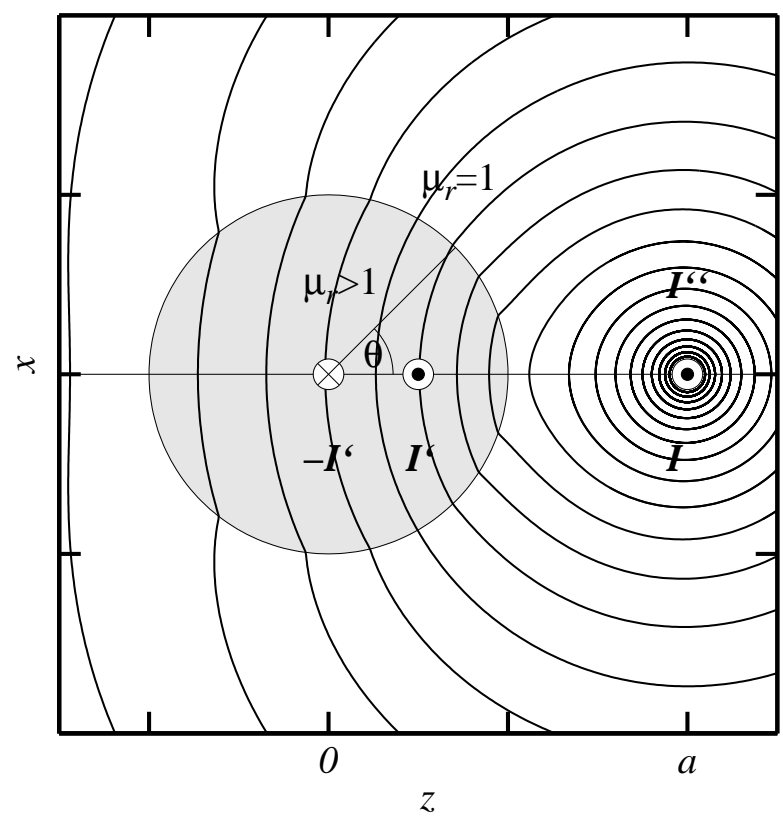

Figure 3: Lines of the magnetic induction $\mathbf{B}$ for a current-carrying straight wire magnetizing a parallel cylinder with magnetic permeability $\mu_{r}=3$. The geometry is identical with fig. 2 .

where $\boldsymbol{e}_{y}$ denotes the unit vector in $y$-direction.

To clarify the physical picture we note that the magnetization $\mathbf{M}=\left(\mu_{r}-1\right) \mathbf{H}$ correspondes to induced bulk currents of density $\boldsymbol{j}_{b}=\nabla \times \mathbf{M}$ and induced surface currents $\boldsymbol{j}_{s}=\mathbf{M} \times \boldsymbol{n}$. In our case $\nabla \times \mathbf{M}=0$ and

$$
j_{s}(\theta)=\frac{I}{\pi} \frac{\mu_{r}-1}{\mu_{r}+1} \frac{a \cos \theta-R}{R^{2}-2 R a \cos \theta+a^{2}} \boldsymbol{e}_{y},
$$

which is analogous to (17). Correspondingly summing up the magnetic forces between these surface currents and the original one we find

$$
F=\frac{\mu_{0} I^{2}}{2 \pi} \frac{\mu_{r}-1}{\mu_{r}+1} \frac{R^{2}}{a\left(a^{2}-R^{2}\right)} .
$$

This result is most easily derived by using (3) which in the present case gives rise to

$$
\mathbf{F}=\mu_{0} \int_{V} d^{3} r(\mathbf{M} \cdot \nabla) \mathbf{H}_{0} .
$$

Exploiting the fact that $\mathbf{M}$ and $\mathbf{H}_{0}$ are parallel it is easy to reproduce (22).

\section{Summary}

Three simple examples have been presented for which the equilibrium force on a polarizable body in an external electromagnetic field can be determined exactly. Different ways to do this have been elucidated. A first possibility is to integrate the Maxwell stress tensor (1) 
over a surface outside the body and completely enclosing it. The second method integrates the volume force density (2) and adds the surface integral over the difference in normal stresses $\left(P_{n}^{2} /\left(2 \varepsilon_{0}\right)+\mu_{0} M_{n}^{2} / 2\right)$ involving the normal components of the polarization and the magnetization. The third and in our opinion most simple and straightforward method builds on the free energy change (3) and uses the formula

$$
\mathbf{F}=\int_{V} d^{3} r\left[(\mathbf{P} \cdot \nabla) \mathbf{E}_{0}+\mu_{0}(\mathbf{M} \cdot \nabla) \mathbf{H}_{0}\right],
$$

where $\mathbf{E}_{0}$ and $\mathbf{H}_{0}$ denote the fields in the absence of the polarizable medium. Note that in the case where the fields do not vary much over the volume of the body this expression reduces to the well known relation

$$
\mathbf{F}=(\mathcal{P} \cdot \nabla) \mathbf{E}_{0}+\mu_{0}(\mathcal{M} \cdot \nabla) \mathbf{H}_{0}
$$

with $\mathcal{P}$ and $\mathcal{M}$ denoting the total polarization and magnetization respectively of the body.

Acknowledgement: We would like to thank Adrian Lange and Hanns-Walter Müller for a critical reading of the manuscript.

a) andreas.engel@physik.uni-magdeburg.de

${ }^{1}$ John D. Jackson, Classical Electrodynamics (John Wiley \& Sons, New York, 1975)

${ }^{2}$ Richard Becker and Fritz Sauter, Theorie der Elektrizität (Teubner, Stuttgart, 1973)

${ }^{3}$ Julius A. Stratton, Electromagnetic Theory (McGraw-Hill, New York, 1941)

${ }^{4}$ L. D. Landau, E. M. Lifshitz, Lehrbuch der theoretischen Physik, Band VIII, Elektrodynamik der Kontinua (Akademie-Verlag, Berlin, 1980)

${ }^{5}$ Stefan Odenbach and Mario Liu, Phys. Rev. Lett. 86, 328 (2001)

${ }^{6}$ comments on ${ }^{5}$, to appear in Phys. Rev. Lett. 